\title{
ON THE SMALL OSCILLATIONS OF THE PERIODIC RAYLEIGH EQUATION*
}

\author{
By
}

HAL L. SMITH

Arizona State University

Introduction. Perturbed Hill's equations arise naturally in the study of many problems in nonlinear oscillations. Stoker [18] observes that "the problem of the infinitesimal stability of the periodic solutions of our nonlinear systems always leads to a Hill's equation." If one is interested in the qualitative behavior of small perturbations of these periodic solutions then one must study not only the linearized Hill's equation but also the effects of the nonlinear terms and damping.

As a motivating physical example consider an electrical circuit consisting of an inductor, a capacitor, and a voltage controlled resistor in parallel. Let $L$ denote the inductance of the coil; assume the capacitance of the capacitor varies periodically with time, $C(t+T)=C(t)$, and the characteristic of the resistor is given by $x=B y^{3}-A y$ where $x$ is the current and $y$ is the voltage. Then the differential equation governing the current through the resistor is

$$
x^{\prime \prime}+\frac{1}{L C(t)}\left(x+B\left(x^{\prime}\right)^{3}-A x^{\prime}\right)=0 .
$$

If we consider only small currents $x$ and assume small damping (positive or negative), $|A|$, in (0.1) then, on neglecting damping and nonlinear effects in (0.1) we obtain a Hill's equation

$$
y^{\prime \prime}+a(t) y=0, a(t) \equiv \frac{1}{L C(t)}=a(t+T) .
$$

An interesting mathematical question is that of the relation between the qualitative behavior of small oscillations of a perturbed Hill's equation, e.g. (0.1) with $|A|$ small, and the qualitative behavior of solutions of the underlying Hill's equation. Since for a set with nonempty interior (in the uniform topology) of $\pi$-periodic, continuous functions $a(t)$, the trivial solution of $(0.2)$ is nonhyperbolic, the effects of small damping and nonlinear terms

*Received December 14, 1983. 
cannot always be neglected. In view of this, can we catalogue the possible qualitative behavior of small oscillations of a perturbed Hill's equation as a function of both the properties of solutions of the underlying Hill's equation and the damping and nonlinear perturbation terms? Some partial answers to this question were given in [16]. The purpose of this paper is to continue this investigation. But rather than consider general nonlinear perturbations and specific "resonant" cases for (0.2) as was done in [16], here we fix the nonlinear terms and consider the gamut of possible behavior of small solutions of a model equation, motivated by the example $(0.1)$, as a function of the behavior of solutions of $(0.2)$. The model equation to be considered is

$$
\begin{gathered}
x^{\prime \prime}+b(t) x^{\prime}\left[\left(x^{\prime}\right)^{2}+\varepsilon\right]+a(t) x=0, \\
\hat{b}=\frac{1}{\pi} \int_{0}^{\pi} b(t) d t \neq 0,
\end{gathered}
$$

in which $\varepsilon$ is a small real parameter and $a(t)$ and $b(t)$ are $\pi$-periodic real-valued continuous functions. The restriction to considering only small damping $\varepsilon$ in $(0.3)$ is motivated by the obvious fact that interesting small oscillations of $(0.3)$ cannot be expected in the presence of large (positive or negative) damping. Indeed, our main interest is in those critical situations where the nonlinear terms together with the damping play a crucial role in determining the possible qualitative behavior of small oscillations. It follows then that for certain resonant cases of the Hill's equation $(0.2)$, the effects of the perturbation terms will be greatest and it will be these cases that will be the focus of this paper.

In order to identify the critical cases, we review here briefly some properties of solutions of (0.2). The most efficient way to describe properties of solutions of $(0.2)$ is in terms of the two Floquet multipliers of $(0.2)$ which are the eigenvalues of the period map (Poincaré map) for the system version of (0.2). It is well known that these multipliers, $\rho_{1}$ and $\rho_{2}$, satisfy $\rho_{1} \cdot \rho_{2}=1$. As a consequence, the only case in which the trivial solution of $(0.2)$ is hyperbolic, that is, when the multipliers lie off the unit circle in the complex plane, occurs when both multipliers are real, of the same sign, and satisfy $\rho_{2}=1 / \rho_{1},\left|\rho_{1}\right|>1$. In this case, a fundamental set for (0.2) will consist of one solution which tends to zero exponentially and another solution which is exponentially unbounded on any half line, i.e., the origin is of saddle type for $(0.2)$. It is well known that saddle type solutions are noncritical with respect to sufficiently small perturbation in the sense that they remain of saddle type. Hence, unless the saddle is very weak, i.e., the multipliers are near to one or minus one, the case that $(0.2)$ is of saddle type is noncritical for solutions of $(0.3)$ and we do not consider it further. Clearly, the remaining case where the trivial solution of $(0.2)$ is nonhyperbolic will be critical for $(0.3)$ in the sense that the perturbation terms in $(0.3)$ can be expected to play a large role in the behavior of small oscillations of $(0.3)$. Both multipliers lie on the unit circle, $\rho_{1}, \rho_{2}=e^{ \pm i \omega \pi}, 0 \leqslant \omega \leqslant 1$. In case $\omega$ is rational, $\omega=p / q$, with $p$ and $q$ relatively prime all solutions of $(0.2)$ will be periodic of period $q \pi$ or $2 q \pi$ depending on whether $p$ is even or odd. It $\omega$ is irrational then all solutions are quasi-periodic with incommensurable periods $\pi$ and $2 \pi / \omega$. 
We shall see that when $\omega \simeq 0,1 / 2,1$, the effect of the nonlinear terms is especially critical in determining the qualitative behavior of small oscillations of $(0.3)$. These are the so-called "strong resonances" $(\omega=1 / 3$ is not present because of the absence of quadratic nonlinearities in (0.3)). In order to model the strong resonances $\omega \simeq 0,1 / 2,1$, we will modify equation $(0.3)$ by writing

$$
a(t)=a_{0}(t)+\delta,
$$

in which $\delta$ is a small real parameter and $a_{0}(t)=a_{0}(t+\pi)$ is such that $(0.2)$ with $a=a_{0}(t)$ has Floquet multipliers $\rho=e^{ \pm i \omega \pi}$ with $\omega=0,1 / 2$ or 1 . One could let $\delta$ be a small norm $\pi$-periodic function as well, but as no new phenomena appear with this complication, we choose the simplest mode of perturbation.

The remaining case where $\omega$ is not near to $0,1 / 2$ or 1 we label as nonresonant. These cases, while critical for $(0.3)$, are not as sensitive to the perturbation terms as are the strongly resonant ones.

We will show that for the nonresonant case there is a Hopf-type bifurcation of an invariant cylinder of solutions of $(0.3)$ in $\left(x, x^{\prime}, t\right)$ space enclosing the trivial solution $(0,0, t)$ which, if we make the identification $\left(x, x^{\prime}, t\right) \sim\left(x, x^{\prime}, t+\pi\right)$, becomes an invariant torus. The stability of this invariant torus depends on whether it bifurcates subcritically or supercritically with respect to the parameter $\varepsilon$ in the usual way. Solutions on the invariant torus can be characterized by the rotation number, $r=r(\varepsilon)$, and regions (in $\varepsilon$ space) of "phase locking" and transition sets of "drifting" solutions are to be expected. All this is fairly standard and predictable on the basis of well-known results concerning one-parameter families of planar diffeomorphisms (see e.g. [11]) and we include a brief description in section 2 for completeness only.

The strongly resonant cases are far more interesting and are the primary focus of this work. They are considered in sections 3 and 4 . While we may expect the possible bifurcation of $\pi, 2 \pi$ or $4 \pi$ periodic subharmonics in the cases $\omega \simeq 0,1,1 / 2$ respectively for $(0.3)$, the existence of invariant cylinders consisting of extremely slowly oscillating solutions surrounding both the trivial solution and the just-mentioned nontrivial subharmonics is perhaps surprising. In addition, there may exist small regions in the $(\varepsilon, \delta)$ plane near the origin in which chaotic solutions exist for $(0.3)$. The chaos is the result of the possible existence of homoclinic or heteroclinic points corresponding to the Poincaré (period) map for (0.3). We cannot prove the existence of these points but genericity considerations strongly suggest their presence. What we can prove in some cases is the negative result that if there is a region of chaos in the $(\varepsilon, \delta)$ plane near the origin, then the region is the envelope of a one-parameter family of curves emanating from the origin which have very high order contact there. From a practical standpoint, the earlier-mentioned existence of extremely slowly oscillating solutions is enough evidence of chaos.

The unifying technique used throughout this paper might be described as simply the method of averaging although we prefer to call it the method of normal forms for periodic systems. It is a natural generalization of the well-known theory of normal forms for autonomous systems $[2,6]$ to periodic systems. In section 1 of this paper we describe that part of the theory which is useful in the present context. A more complete account of the 
theory can be found in [2] or [17]. In the present context, the method consists of a sequence of near-identity transformations in a system formulation of $(0.3)$, producing an equivalent system which is autonomous to some order in the variables $\left(x, x^{\prime}, \varepsilon\right)$ (or $\left.\left(x, x^{\prime}, \varepsilon, \delta\right)\right)$. The higher-order terms are $\pi$-periodic. Ignoring the higher-order terms, we find that the phase portraits of the resulting autonomous systems have already been worked out in the literature. One must then determine the effects of the higher-order, $\pi$-periodic perturbation terms which are not explicitly known. Standard theorems from the theory of ordinary differential equations can be used to determine the effects of perturbation when the phase portrait of the autonomous system is structurally stable. The so-called Melnikov Theory [13, 5, 9] is used to determine qualitatively the effect of perturbation on saddle-connecting orbits of the planar system although our lack of an explicit formula for the lowest-order perturbation terms limits our treatment of these cases.

None of the tools used in this work are new and all the analyses of planar autonomous systems which arise in this work appear in the literature. We view the essential contribution made in this paper as being the explicit calculation of the coefficients of the various normal forms in terms of the perturbation terms in $(0.3)$ and certain solutions of the Hill's equation (0.2). In principle, this allows the prediction of which of several possible bifurcation sequences actually occurs in a given system by numerical or analytical calculations involving only the linear Hill's equation. In addition, as it appears that the normal form theory used here is not well known, perhaps this work will serve to point out its effectiveness and some of its deficiencies in analysing concrete problems in nonlinear oscillations.

1. Normal forms. In this section we briefly describe the application of the theory of normal forms to the problem of small oscillations of $(0.3)$. The reader interested in a more complete description of the theory may consult [2] (See also [17]). We may write (0.3) in system form as

$$
\hat{x}^{\prime}=A_{0}(t) \hat{x}+\varepsilon A_{1}(t) \hat{x}+Q_{3}(t)[\hat{x}]^{3}, \quad \hat{x}=\operatorname{col}\left(x, x^{\prime}\right),
$$

in which

$$
A_{0}(t)=\left(\begin{array}{cc}
0 & 1 \\
-a(t) & 0
\end{array}\right), \quad A_{1}(t)=\left(\begin{array}{cc}
0 & 0 \\
0 & -b(t)
\end{array}\right)
$$

and the homogeneous cubic term, denoted by $Q_{3}(t)[\hat{x}]^{3}$, is

$$
Q_{3}(t)[\hat{x}]^{3}=\left(\begin{array}{c}
0 \\
-b\left(x^{\prime}\right)^{3}
\end{array}\right) .
$$

The unperturbed Hill's equation

$$
\hat{x}^{\prime}=A_{0}(t) \hat{x}
$$

has, by the Floquet Theory, a fundamental matrix solution

$$
\Phi(t)=P(t) e^{A t}, \quad P(t+\pi)=P(t),
$$


where the eigenvalues of $A$ are the Floquet exponents. We will take particular care in our selection of $\Phi(t)$ so that $A$ will be in canonical form and $P(t)$ will be real, although this is not crucial.

The Floquet change of variables

$$
\hat{x}=P(t) \bar{x}
$$

in $(1.1)$ yields

$$
\bar{x}^{\prime}=A \bar{x}+\varepsilon \tilde{A_{1}}(t) \bar{x}+\tilde{Q}_{3}(t)[\bar{x}]^{3}
$$

in which

$$
\begin{aligned}
& \tilde{A}_{1}(t)=P^{-1} A_{1} P, \\
& \tilde{Q}_{3}(t)[\bar{x}]^{3}=P^{-1} Q_{3}(t)[P \bar{x}]^{3} .
\end{aligned}
$$

The Floquet change of variables effectively makes (1.3) autonomous to first-order terms in $(\bar{x}, \varepsilon)$. We now seek a transformation of variables of the form

$$
\bar{x}=z+\varepsilon M_{1}(t) z
$$

where the matrix $M_{1}(t), \pi$-periodic in $t$, is to be determined so that the resulting equation for $z$ is autonomous to second-order terms in $(z, \varepsilon)$. Substitution of (1.4) into (1.3) leads to

$$
\begin{aligned}
z^{\prime}= & A z+\varepsilon\left[\tilde{A}_{1}-\left(M_{1}^{\prime}+M_{1} A-A M_{1}\right)\right] z \\
& +\varepsilon^{2} B(t) z+\tilde{Q}_{3}(t)[z]^{3}+O\left((|z|+|\varepsilon|)^{4}\right) .
\end{aligned}
$$

A linear term in $z$ of order $\varepsilon^{2}$ is introduced by the transformation (1.4). We will need its explicit form

$$
B(t)=\tilde{A_{1}} M_{1}-M_{1}\left[\tilde{A_{1}}-\left(M_{1}^{\prime}+M_{1} A-A M_{1}\right)\right]
$$

later in our calculation. The idea now is to select $M_{1}(t)$ in such a way that the first bracket in (1.5) simplifies as much as possible. The simplification that can be made in this term will depend crucially on the eigenvalues (Floquet exponents of (0.2)) of $A$.

The form of the order $\varepsilon$ terms in (1.5) suggests the consideration of the matrix equation

$$
M_{1}^{\prime}+M_{1} A-A M_{1}=\phi(t)=\phi(t+\pi)
$$

where $\phi(t)$ is a $\pi$-periodic $2 \times 2$ matrix. One seeks to determine the subspace of $\pi$-periodic $2 \times 2$ matrix functions $\phi$ for which (1.7) has a $\pi$-periodic solution $M_{1}$, that is, one seeks to determine the range of the differential operator on the left of (1.7) acting on the $\pi$-periodic matrix-valued functions. If $\tilde{A}_{1}$ lies in this range, then $M_{1}$ can be selected so that the order $\varepsilon$ terms in (1.5) vanish ( $M_{1}$ would be a solution of (1.7) with $\phi=\tilde{A}_{1}$ ). In general, $\tilde{A}_{1}$ will not lie in the range but one can write

$$
\tilde{A_{1}}=\tilde{A_{1,1}}+\tilde{A_{1,2}}
$$

where both $\tilde{A}_{1, j}$ are $\pi$-periodic, $j=1,2, \tilde{A_{1,1}}$ lies in the range and $\tilde{A}_{1,2}$ lies in some complementary subspace to the range. Clearly, one can then select $M_{1}$ so that the order $\varepsilon$ terms in (1.5) become $\tilde{A}_{1,2}\left(M_{1}\right.$ is a solution of (1.7) with $\left.\phi=\tilde{A}_{1,1}\right)$. An important point to notice is that $\tilde{A}_{1,2}$ is not uniquely determined, it depends on the selection of a subspace complementary to the range. In many applications $\tilde{A}_{1,2}$ will be independent of $t$ or have at most one frequency, greatly simplifying (1.5). 
Using only the well-known fact that the scalar equation

$$
x^{\prime}(t)=f(t)=f(t+\pi)
$$

has a $\pi$-periodic solution $x(t)$ if and only if

$$
[f] \equiv \frac{1}{\pi} \int_{0}^{\pi} f(t) d t=0,
$$

the reader may easily verify the entries in the last column of Table 1.1 .

TABLE 1.1. Normal forms for matrices

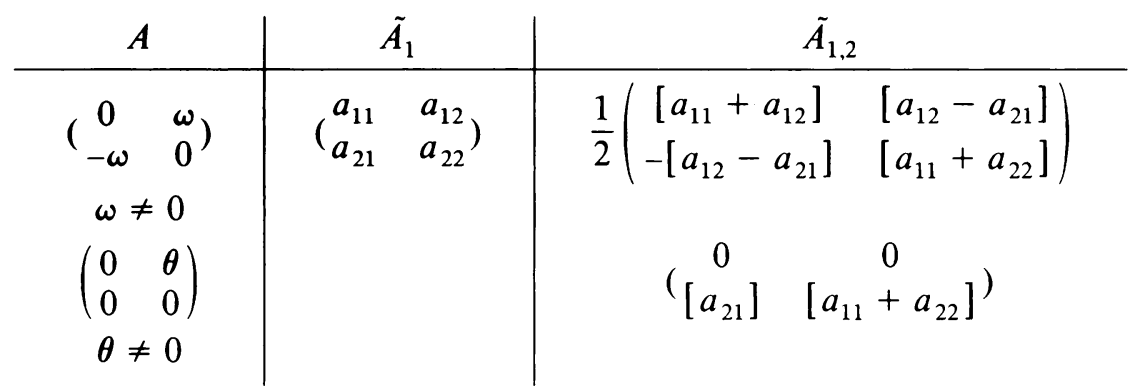

In fact, if

$$
M_{1}=\left(\begin{array}{ll}
m_{11} & m_{12} \\
m_{21} & m_{22}
\end{array}\right)
$$

and $A$ is as in the top of Table 1.1, then (1.7) becomes

$$
\begin{aligned}
& m_{11}^{\prime}+\omega\left(m_{12}+m_{21}\right)=\phi_{11}, \\
& m_{12}^{\prime}-\omega\left(m_{11}-m_{22}\right)=\phi_{12}, \\
& m_{21}^{\prime}-\omega\left(m_{11}-m_{22}\right)=\phi_{21} \\
& m_{22}^{\prime}-\omega\left(m_{21}+m_{12}\right)=\phi_{22}
\end{aligned}
$$

Defining

$$
\begin{aligned}
& v_{1}=m_{11}+m_{22}, \\
& v_{2}=m_{12}-m_{21}, \\
& v_{3}=m_{11}-m_{22}, \\
& v_{4}=m_{12}+m_{21},
\end{aligned}
$$

the resulting equations for $v$ become

$$
\begin{aligned}
& v_{1}^{\prime}=\phi_{11}+\phi_{22}, \\
& v_{2}^{\prime}=\phi_{12}-\phi_{21}, \\
& v_{3}^{\prime}+2 \omega v_{4}=\phi_{11}-\phi_{22}, \\
& v_{4}^{\prime}-2 \omega v_{3}=\phi_{12}+\phi_{21} .
\end{aligned}
$$

It follows from these equations that the range of the differential operator on the right of (1.7) consists of those $\pi$-periodic $2 \times 2$ matrix functions $\phi$ having

$$
\left[\phi_{11}+\phi_{22}\right]=\left[\phi_{12}-\phi_{21}\right]=0 \text {. }
$$


Hence, we may decompose $\tilde{A_{1}}$ as

$$
\tilde{A_{1}}=\left(\tilde{A_{1}}-\tilde{A_{1,2}}\right)+\tilde{A_{1,2}}
$$

where $\tilde{A_{1,2}}$ is as in Table 1.1 and $\tilde{A_{1}}-\tilde{A_{1,2}}$ lies in the range.

The particular $A$ in Table 1.1 are those which arise naturally in our later study of (1.1). Notice that in either case, $\tilde{A}_{1,2}$ is independent of $t$ so that (1.5) can be made autonomous through second-order terms in $(z, \varepsilon)$ :

$$
z^{\prime}=A z+\varepsilon \tilde{A_{1,2}} z+\varepsilon^{2} B(t) z+\tilde{Q}_{3}(t)[z]^{3}+O\left((|z|+|\varepsilon|)^{4}\right) .
$$

Finally, a change of variables of the form

$$
z=w+\varepsilon^{2} M_{2}(t) w+D_{3}(t)[w]^{3}
$$

is sought, in which $M_{2}(t)$ is a $\pi$-periodic matrix and $D_{3}(t)[w]^{3}$ is a homogeneous cubic polynomial in each component with coefficients that are $\pi$-periodic in $t$, so that the cubic terms in $(w, \varepsilon)$ in the resulting equation for $w$ have as simple a form (autonomous, hopefully) as possible. Substitution of (1.9) into (1.8) (note that $B(t)=\tilde{A_{1}} M_{1}-M_{1} \tilde{A_{1,2}}$ ) yields the differential equation for $w$,

$$
\begin{aligned}
w^{\prime}= & A w+\varepsilon \tilde{A_{1,2} w}+\varepsilon^{2}\left[B-\left(M_{2}^{\prime}+M_{2} A-A M_{2}\right)\right] w \\
& +\left[\tilde{Q}_{3}(t)[w]^{3}-\left(D_{3}^{\prime}(t)[w]^{3}+\frac{\partial}{\partial w}\left(D_{3}(t)[w]^{3}\right) A w-A D_{3}(t)[w]^{3}\right)\right. \\
& +O\left((|w|+|\varepsilon|)^{4}\right) .
\end{aligned}
$$

Clearly, $M_{2}$ can be selected in the same fashion as $M_{1}$. However, since $B(t)$ depends on $M_{1}(t)$, the explicit form of the term in the first bracket of (1.10) after appropriate selection of $M_{2}$, will be more complicated. Our interest here is primarily the elucidation of the qualitative properties of solutions and for this, explicit knowledge of the second-order correction to the linear part of the vector field is not crucial.

The cubic terms in the second bracket in (1.10) can be treated in the same way as the linear terms in $w$. The equation to consider is

$$
D_{3}^{\prime}+L D_{3}=\psi(t)=\psi(t+\pi)
$$

where $L$ is the linear operator on the space of homogeneous cubic polynomials given by

$$
L D_{3}=\left(\frac{\partial}{\partial z} D_{3}\right) A z-A D_{3}
$$

and $\psi$ is a homogeneous cubic polynomial (in each component) with $\pi$-periodic coefficients. The operator " $(\partial / \partial t+L)$ " on the left of (1.11), acting on the homogeneous cubic polynomials with $\pi$-periodic coefficients, has a range and $D_{3}$ can be selected so that the component of $\tilde{Q}_{3}$ along the range can be eliminated from the cubic terms in (1.10). In computations it is convenient to employ the following representation of a homogeneous cubic polynomial

$$
\left(q_{11}, q_{12}, q_{13}, q_{14}, q_{21}, q_{22}, q_{23}, q_{24}\right)=\left(\begin{array}{c}
q_{11} \bar{X}_{1}^{3}+q_{12} \bar{X}_{1}^{2} \bar{X}_{2}+q_{12} \bar{X}_{1} \bar{X}_{2}^{2}+q_{14} \bar{X}_{2}^{3} \\
q_{21} \bar{X}_{1}^{3}+q_{22} \bar{X}_{1}^{2} \bar{X}_{2}+q_{23} \bar{X}_{1} \bar{X}_{2}^{2}+q_{24} \bar{X}_{2}^{3}
\end{array}\right)
$$


Using this notation, (1.11) becomes a first order linear inhomogeneous system with constant coefficients. Again, the reader will find it a straightforward exercise (though somewhat tedious) to verify Table 1.2 . (We write $\tilde{Q}_{3}=\tilde{Q}_{3,1}+\tilde{Q}_{3,2}$, where $\tilde{Q}_{3,1}$ lies in the range of (1.11).)

TABLE 1.2. Normal forms for cubics

\begin{tabular}{|c|c|c|}
\hline$A$ & $\tilde{Q}_{3}$ & $\tilde{Q}_{3,2}$ \\
\hline $\begin{array}{c}\left(\begin{array}{cc}0 & \omega \\
-\omega & 0\end{array}\right) \\
0<\omega<1 \\
\omega \neq 1 / 2\end{array}$ & $\left(\begin{array}{l}q_{11} \bar{x}_{1}^{3}+q_{12} \bar{x}_{1}^{2} \bar{x}_{2}+q_{13} \bar{x}_{1} \bar{x}_{2}^{2}+q_{14} \bar{x}_{2}^{3} \\
q_{21} \bar{x}_{1}^{3}+q_{22} \bar{x}_{1}^{2} \bar{x}_{2}+q_{23} \bar{x}_{1} \bar{x}_{2}^{2}+q_{24} \bar{x}_{2}^{3}\end{array}\right)$ & $\begin{array}{c}r^{2}\left(\begin{array}{cc}\alpha & \beta \\
-\beta & \alpha\end{array}\right) z \\
r^{2}=z \cdot z \\
\alpha=\frac{1}{8}\left(3\left[q_{11}\right]+\left[q_{13}\right]+\left[q_{22}\right]+3\left[q_{24}\right]\right) \\
\beta=-\frac{1}{8}\left(\left[3 q_{21}+q_{23}-q_{12}-3 q_{14}\right]\right)\end{array}$ \\
\hline$\left(\begin{array}{cc}0 & 1 / 2 \\
-\frac{1}{2} & 0\end{array}\right)$ & & $\begin{array}{c}r^{2}\left(\begin{array}{cc}\alpha & \beta \\
-\beta & \alpha\end{array}\right) z+ \\
\left(z_{1}^{3}-3 z_{1} z_{2}^{2}\right)\left(\begin{array}{c}\Lambda_{1}(t) \\
-\Lambda_{2}(t)\end{array}\right)+ \\
\left(z_{2}^{3}-3 z_{1}^{2} z_{2}\right)\left(\begin{array}{c}\Lambda_{2}(t) \\
\Lambda_{1}(t)\end{array}\right) \\
\Lambda(t)=\Lambda_{1}+i \Lambda_{2}=\lambda \mathrm{e}^{2 i t} \\
\lambda=\frac{1}{8}\left[\left\langle\tilde{Q}_{3}(t), \bar{n}\right\rangle\right] \\
n=e^{-2 i t}(1,-i,-1, i,-i,-1, i, 1) \\
\langle\cdot, \cdot\rangle=\text { inner product on } \mathbf{C}^{8}\end{array}$ \\
\hline $\begin{array}{c}\left(\begin{array}{ll}0 & \theta \\
0 & 0\end{array}\right) \\
\theta \neq 0\end{array}$ & & $\left(\begin{array}{c}0 \\
{\left[q_{21}\right] z_{1}^{3}+\left[q_{22}+3 q_{11}\right] z_{1}^{2} z_{2}}\end{array}\right)$ \\
\hline
\end{tabular}

The nonautonomous term in the case $\omega=1 / 2$ is due to the fact that the homogeneous equation adjoint to (1.11) has not only a zero eigenvalue (as in the case $\omega \neq 1 / 2$ ) but also the complex conjugate pair $\pm 2 i$ resulting in the $\pi$-periodic solutions $n(t)$ and $\bar{n}(t)$ as in Table 1.2. See $[16,17]$ for further details concerning the calculations in Tables 1.1 and 1.2.

The net effect of the successive transformations (1.2), (1.4), and (1.9) is the composite transformation

$$
\hat{x}=P(t)\left[w+\varepsilon M_{1}(t) w+\varepsilon^{2} M_{2}(t) w+D_{3}(t)[w]^{3}+\ldots\right]
$$

which transforms (1.1) to (assuming $A$ as in Tables 1.1 and 1.2)

$$
w^{\prime}=A w+\varepsilon \tilde{A_{1,2}} w+\varepsilon^{2} B_{1,2} w+\tilde{Q}_{3,2}(t)[w]^{3}+O\left((|\varepsilon|+|w|)^{4}\right),
$$

in which terms of order four and greater are $\pi$-periodic in $t$. Ignoring the higher-order terms in (1.13), we obtain the equation

$$
w^{\prime}=A w+\varepsilon \tilde{A}_{1,2} w+\varepsilon^{2} B_{1,2} w+\tilde{Q}_{3,2}(t)[w]^{3} .
$$

We will refer to (1.14) as the normal form of (1.1) through terms of order four. 
2. The nonresonant cases. In this section we consider $(0.3)$ when the reduced Hill's equation $(0.2)$ is nonresonant. In this case, the Floquet multipliers of $(0.2)$ lie on the unit circle but not near the real or imaginary axis and we denote them by $\rho_{ \pm}=e^{ \pm i \omega \pi}, 0<\omega<$ $1, \omega \neq 1 / 2$. By Floquet theory, (0.2) has a nontrivial solution of the form

$$
y(t)=e^{i \omega t} q(t), \quad q(t+\pi)=q(t)=u+i v .
$$

It is easy to verify that, if $y=y_{1}+i y_{2}$, then $y_{1}$ and $y_{2}$ make up a fundamental set of solutions of (0.2). Clearly, $y(t)$ can be further chosen so that the Wronskian, $W\left(y_{1}, y_{2}\right)(t)$ $=y_{1}^{\prime} y_{2}-y_{2}^{\prime} y_{1}$, is either +1 or -1 by the multiplication of $y$ by a constant. In case $W\left(y_{1}, y_{2}\right) \equiv+1$ we have a fundamental matrix $\Phi$ given by

$$
\Phi(t)=\left(\begin{array}{ll}
y_{1} & y_{2} \\
y_{1}^{\prime} & y_{2}^{\prime}
\end{array}\right)=P(t) \exp \left(\begin{array}{cc}
0 & \omega \\
-\omega & 0
\end{array}\right) t,
$$

in which

$$
P(t)=\left(\begin{array}{cc}
u & v \\
\bar{u} & \bar{v}
\end{array}\right)
$$

where $\bar{u}=u^{\prime}-\omega v, \bar{v}=v^{\prime}+\omega u$. It follows that

$$
P(t+\pi)=P(t) \text { and } \operatorname{det} P(t) \equiv 1 .
$$

In case $W\left(y_{1}, y_{2}\right)=-1$ we take

$$
\Phi(t)=\left(\begin{array}{ll}
y_{1} & -y_{2} \\
y_{1}^{\prime} & -y_{2}^{\prime}
\end{array}\right)=P(t) \exp \left(\begin{array}{cc}
0 & -\omega \\
\omega & 0
\end{array}\right) t,
$$

in which

$$
P(t)=\left(\begin{array}{ll}
u & -v \\
\bar{u} & -\bar{v}
\end{array}\right)
$$

and (2.1) holds. We hereafter assume $W\left(y_{1}, y_{2}\right)=+1$.

Making the Floquet transformation (1.2) with $P(t)$ given by (2.2) leads to

$$
y^{\prime}=\omega\left(\begin{array}{cc}
0 & +1 \\
-1 & 0
\end{array}\right) y+\varepsilon \tilde{A_{1}}(t) y+\tilde{Q}_{3}(t)[y]^{3},
$$

in which

$$
\begin{gathered}
\tilde{A}_{1}(t)=-b\left(\begin{array}{cc}
-u \bar{v} & -v \bar{v} \\
u \bar{u} & u \bar{v}
\end{array}\right), \\
\tilde{Q}_{3}(t)[y]^{3}=b\left(\begin{array}{c}
v\left(\bar{u} y_{1}+\bar{v} y_{2}\right)^{3} \\
-u\left(\bar{u} y_{1}+\bar{v} y_{2}\right)^{3}
\end{array}\right) .
\end{gathered}
$$

From (1.14) and Tables 1.1 and 1.2, it follows immediately that the normal form for equation $(0.3)$ in the case that $(0.2)$ is nonresonant, is given by

$$
w^{\prime}=\left(\begin{array}{ll}
\varepsilon \gamma_{1}+\varepsilon^{2} \gamma_{2} & \omega+\varepsilon \zeta_{1}+\varepsilon^{2} \zeta_{2} \\
-\omega-\varepsilon \zeta_{1}-\varepsilon^{2} \zeta_{2} & \varepsilon \gamma_{1}+\varepsilon^{2} \gamma_{2}
\end{array}\right) w+r^{2}\left(\begin{array}{cc}
\alpha & \beta \\
-\beta & \alpha
\end{array}\right) w .
$$

In polar form, the equation can be written as

$$
\begin{aligned}
& r^{\prime}=r\left(\varepsilon \gamma_{1}+\varepsilon^{2} \gamma_{2}+\alpha r^{2}\right), \\
& \theta^{\prime}=-\omega-\varepsilon \zeta_{1}-\varepsilon^{2} \zeta_{2}-\beta r^{2},
\end{aligned}
$$


in which

$$
\begin{aligned}
& \gamma_{1}=-\hat{b} / 2, \\
& \zeta_{1}=\frac{1}{2} \operatorname{Re}\left[b \bar{y} y^{\prime}\right], \\
& \alpha=-\frac{3}{8}\left[b\left|y^{\prime}\right|^{2}\right], \\
& \beta=\frac{3}{8} \operatorname{Re}\left[b\left|y^{\prime}\right|^{2} y^{\prime} \bar{y}\right] .
\end{aligned}
$$

Explicit formulae can be given for $\gamma_{2}$ and $\zeta_{2}$ in terms of $b, y, y^{\prime}$. They are not particularly enlightening. We merely note that from Table 1.1 applied to $B(t)=\tilde{A}_{1}(t) M_{1}(t)-$ $M_{1}(t) \tilde{A_{1,2}}$,

$$
\gamma_{2}=\frac{1}{2}[\operatorname{tr} B(t)], \quad \zeta_{2}=-\frac{1}{2}[\operatorname{trJ} B(t)], \quad J=\left(\begin{array}{cc}
0 & 1 \\
-1 & 0
\end{array}\right)
$$

In Fig. 2.1 the phase portraits of (2.7) are given in the case that $\hat{b}>0$ and $\alpha \neq 0$. The essential feature of the phase portrait in both cases $\alpha<0$ and $\alpha>0$ is the occurrence of a Hopf bifurcation from the trivial solution as $\varepsilon$ passes through zero. The bifurcating periodic orbit is subcritical and unstable if $\alpha>0$ and supercritical and stable if $\alpha<0$. Note that the sign of $\alpha$ is determined by the function $b(t)$ together with $\left|y^{\prime}(t)\right|^{2}$ where $y(t)$ is the special solution of (0.2) given in (2.1). Any complex multiple of $y(t)$ will not change this sign, it depends only on the subspace spanned by $y(t)$.

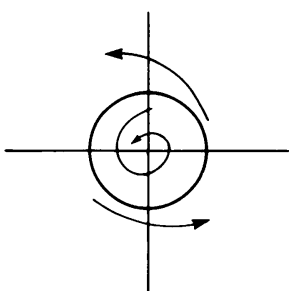

a) $\alpha>0, \varepsilon>0$

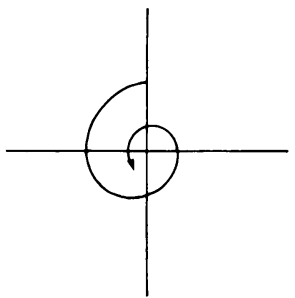

c) $\alpha<0, \varepsilon>0$

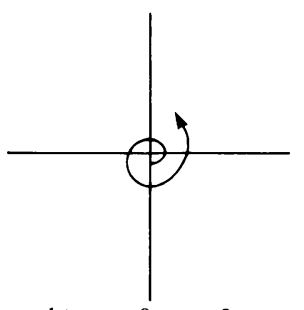

b) $\alpha>0, \varepsilon<0$

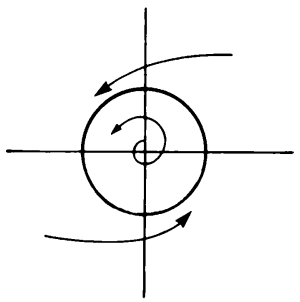

d) $\alpha<0$, $\varepsilon<0$

FIG. 2.1. Phase portraits of (2.7), $\hat{b}>0$ 
Equation (2.7) is accurate to the cubic terms in $(r, \varepsilon)$, the truncated terms being $\pi$-periodic in $t$ and vanishing when $r=0$. It follows from well-known results for ordinary differential equations that for $\varepsilon \neq 0$ and sufficiently small, the trivial solution of (2.7) with the higher-order terms added on has the same stability type as depicted in Fig. 2.1. The Hopf bifurcation of a periodic orbit for (2.7) signals the bifurcation of an invariant cylinder of solutions of the periodically perturbed equation (2.7). This invariant cylinder has the same stability properties enjoyed by the invariant cylinder which the periodic orbits in Figure (2.1) become when the extra dimension of $t$ is added. In order to see the implications of these conclusions for equation (0.3), recall that from (1.12) we have the approximate formula

$$
x(t)=r(t)[u(t) \cos \theta(t)+v(t) \sin \theta(t)]+O\left((|r|+|\varepsilon|)^{2}\right),
$$

in which $u$ and $v$ are $\pi$-periodic in $t$. It is not difficult to see that the invariant cylinder of solutions of the periodically perturbed version of (2.7) gives rise to an invariant cylinder of solutions of $(0.3)$ enclosing the trivial solution $(0,0, t)$ having the form

$$
S_{\varepsilon}=\left\{\left(x, x^{\prime}, t\right):\left(x, x^{\prime}\right)=\eta(\psi, t, \varepsilon) P(t)\left(\begin{array}{c}
\cos \psi \\
\sin \psi
\end{array}\right), 0 \leqslant \psi \leqslant 2 \pi, t \in R\right\}
$$

in which $\eta(\psi+2 \pi, t, \varepsilon)=\eta(\psi, t+\pi, \varepsilon)=\eta(\psi, t, \varepsilon)$ has the asymptotic representation

$$
\eta(\psi, t, \varepsilon)=\sqrt{\frac{-\varepsilon \gamma_{1}}{\alpha}}+O(\varepsilon)
$$

uniformly in $(\psi, t)$.

Since $\eta$ is $\pi$-periodic in $t, S_{\varepsilon}$ can be viewed as a torus on the identification $\left(x, x^{\prime}, t+\pi\right)$ $\sim\left(x, x^{\prime}, t\right)$. The behavior of solutions on $S_{\varepsilon}$ can be approximately described by the scalar equation

$$
\psi^{\prime}=-\omega-\varepsilon\left(\zeta_{1}+\beta \frac{\gamma_{1}}{\alpha}\right)+O\left(\varepsilon^{2}\right),
$$

in which the $O\left(\varepsilon^{2}\right)$ terms are $2 \pi$-periodic in $\psi$ and $\pi$-periodic in $t$. More precisely, if we consider the Poincare map (period $\pi$ map) restricted to the circle $C_{\varepsilon}=S_{\varepsilon} \cap\{t=0\}$ as a map onto $C_{\varepsilon}$, we obtain a one-parameter family of circle maps parametrized by $\varepsilon>0$ in case $\alpha>0$ or $\varepsilon<0$ in case $\alpha<0$. From known genericity results concerning one-parameter families of circle maps ([8]) we can expect zones (open sets) of values of $\varepsilon$ for which solutions on the invariant cylinder are asymptotic to one of a finite number of $m \pi$-periodic solutions for a fixed $m, m / n \approx 2 / \omega$ for an integer $n$ (depending on the zone). This is commonly referred to as phase locking. These zones are separated by transition (closed) sets for which solutions on the invariant cylinder, viewed as a torus, wind densely around the torus.

3. Near resonance: $\omega \approx 0,1 / 2$, or 1 . In this section, $(0.3)$ is considered with $a(t)$ given by ( 0.4$)$ and $\delta=0$ corresponding to one of the following resonance conditions: (i) both Floquet multipliers are +1 and $(0.2)$ has a one-dimensional space of $\pi$-periodic solutions; (ii) both Floquet multipliers are -1 and $(0.2)$ has a one-dimensional space of $2 \pi$-periodic solutions; (iii) the Floquet multipliers are $\pm i$ and $(0.3)$ has a two-dimensional space of $4 \pi$-periodic solutions. 
The restriction in cases (i) and (ii) to a one-dimensional space of $\pi$ or $2 \pi$-periodic solutions for the reduced Hill's equation is motivated by the fact that this is the generic case [15]. We consider case (i) and (ii) together in Section $3 \mathrm{~A}$ immediately below and refer to these two cases in terms of their Floquet multipliers, that is, (i) will be referred to by " $\rho=+1$ " and (ii) by " $\rho=-1$ ". Case (iii) will be considered later in section 3B.

$3 \mathrm{~A} . \omega \approx 0$ or 1 . It will be useful to review here some elementary results from the theory of Hill's equations. More details can be found in $[12,7]$. In system form, $(0.2)$ with $(0.4)$ becomes

$$
\left(\begin{array}{l}
y \\
y^{\prime}
\end{array}\right)^{\prime}=\left(\begin{array}{cc}
0 & 1 \\
-a_{0}(t)-\delta & 0
\end{array}\right)\left(\begin{array}{l}
y \\
y^{\prime}
\end{array}\right) .
$$

Let $I$ denote the two-by-two identity matrix and $\psi_{\delta}(t), \psi_{\delta}(0)=I$ be the fundamental matrix for (3.1). The Floquet multipliers of (3.1) are the roots of the quadratic equation

$$
\rho^{2}-2 \Delta(\delta) \rho+1=0, \quad \Delta(\delta)=\operatorname{trace} \psi_{\delta}(\pi)
$$

The two cases considered in this section are characterized by $\Delta(0)=\rho, \Delta^{\prime}(0) \neq 0$. The condition $\Delta^{\prime}(0) \neq 0$ implies that $\delta=0$ is a transition value between two different stability types. If $\Delta^{\prime}(0) \rho>0$ then (3.1) is nonresonant for $\delta<0$ and of saddle type for $\delta>0$. The reverse inequality reverses the stability types.

Floquet theory implies the existence of a solution of $(0.2)$ for $\delta=0$ satisfying

$$
y(t+\pi)=\rho y(t) .
$$

Let $\tilde{y}(t)$ be another solution such that the Wronskian, $W(y, \tilde{y})=+1$. Such a pair of solutions can always be found. It follows from the Floquet theory [12] that there exists a nonzero real number $\theta$ for which

$$
\tilde{y}(t+\pi)=\rho(\tilde{y}(t)+\pi \theta y(t)), \quad t \in R .
$$

The fundamental matrix solution of (3.1) for $\delta=0$, defined by

$$
\Phi(t)=\left(\begin{array}{cc}
y & \tilde{y} \\
y^{\prime} & \tilde{y}^{\prime}
\end{array}\right)
$$

satisfies

$$
\Phi(t)=P(t) \exp \left(\begin{array}{ll}
0 & \theta \\
0 & 0
\end{array}\right) t
$$

where

$$
P(t)=\left(\begin{array}{ll}
y & \bar{y}_{1} \\
y^{\prime} & \bar{y}_{2}
\end{array}\right)=\rho P(t+\pi)
$$

and

$$
\begin{aligned}
& \bar{y}_{1}(t)=\tilde{y}(t)-\theta t y(t), \\
& \bar{y}_{2}(t)=\tilde{y}^{\prime}(t)-\theta t y^{\prime}(t) .
\end{aligned}
$$


It turns out ([16]) that $\theta$ and $\Delta^{\prime}(0)$ are related by

$$
\theta=-\rho \frac{2 \Delta^{\prime}(0)}{\pi^{2}\left[y^{2}\right]} .
$$

We now turn our attention to the nonlinear equation (0.3). Our discussion of normal forms in Sec. 1 did not take account of the introduction of a second small parameter, $\delta$, in (0.3). The presence of another small parameter hardly affects the analysis in Sec. 1, however, so we proceed exactly as in that section. Write $(0.3),(0.4)$ in system form

$$
\hat{x}^{\prime}=A_{0}(t) \hat{x}+\delta A_{1}(t) \hat{x}+\varepsilon A_{2}(t) \hat{x}+Q_{3}(t)[\hat{x}]^{3},
$$

in which $\hat{x}=\left(x, x^{\prime}\right)$ and

$$
\begin{gathered}
A_{0}(t)=\left(\begin{array}{cc}
0 & 1 \\
-a_{0}(t) & 0
\end{array}\right), \\
A_{1}(t)=\left(\begin{array}{cc}
0 & 0 \\
-1 & 0
\end{array}\right), \\
A_{2}(t)=\left(\begin{array}{cc}
0 & 0 \\
0 & -b(t)
\end{array}\right), \\
Q_{3}(t)[\hat{x}]^{3}=\left(\begin{array}{l}
0 \\
-b(t)\left(x^{\prime}\right)^{3}
\end{array}\right) .
\end{gathered}
$$

Making the Floquet change of variables (1.2) in (3.2) results in

$$
\bar{x}^{\prime}=\left(\begin{array}{ll}
0 & \theta \\
0 & 0
\end{array}\right) \bar{x}+\delta \tilde{A_{1}}(t) \bar{x}+\varepsilon \tilde{A}_{2}(t) \bar{x}+\tilde{Q}_{3}(t)[\bar{x}]^{3},
$$

in which

$$
\begin{gathered}
\tilde{A}_{1}(t)=\left(\begin{array}{cc}
y \bar{y}_{1} & \bar{y}_{1}^{2} \\
-y^{2} & -\bar{y}_{1} y
\end{array}\right), \\
\tilde{A}_{2}(t)=b(t)\left(\begin{array}{cc}
y^{\prime} \bar{y}_{1} & \bar{y}_{1} \bar{y}_{2} \\
-y y^{\prime} & -\bar{y}_{2} y
\end{array}\right), \\
\tilde{Q}_{3}(t)[\bar{x}]^{3}=b(t)\left(\begin{array}{l}
\bar{y}_{1}\left(\bar{x}_{1} y^{\prime}+\bar{x}_{2} \bar{y}_{2}\right)^{3} \\
-y\left(\bar{x}_{1} y^{\prime}+\bar{x}_{2} \bar{y}_{2}\right)^{3}
\end{array}\right) .
\end{gathered}
$$

It is important to observe that in both cases, $\rho= \pm 1$, all functions in (3.3) are $\pi$-periodic.

Following Sec. 1, we make the change of variables in (3.3),

$$
\bar{x}=z+\delta M_{1}(t) z+\varepsilon M_{2}(t) z
$$

where the $\pi$-periodic $2 \times 2$ matrices $M_{i}(t)$ can be chosen as in Table 1.1 so as to make the differential equation for $z$ autonomous through quadratic terms in $(z, \delta, \varepsilon)$. We obtain

$$
\begin{aligned}
z^{\prime}= & \left(\begin{array}{ll}
0 & \theta \\
0 & 0
\end{array}\right) z+\delta\left(\begin{array}{cc}
0 & 0 \\
\bar{\gamma}_{1} & 0
\end{array}\right) z+\varepsilon\left(\begin{array}{cc}
0 & 0 \\
\bar{\gamma}_{2} & \gamma_{3}
\end{array}\right) z \\
& +\delta^{2} B_{1}(t) z+\varepsilon \delta B_{2}(t) z+\varepsilon^{2} B_{3}(t) z+\tilde{Q}_{3}(t)[z]^{3} \\
& +O\left((|z|+|\delta|+|\varepsilon|)^{4}\right),
\end{aligned}
$$


in which

$$
\begin{aligned}
& \bar{\gamma}_{1}=-\left[y^{2}\right] \\
& \bar{\gamma}_{2}=-\left[b y y^{\prime}\right] \\
& \gamma_{3}=-\hat{b} \\
& B_{1}(t)=\tilde{A_{1}} M_{1}-M_{1} \tilde{A}_{1,2} \\
& B_{3}(t)=\tilde{A}_{2} M_{2}-M_{2} \tilde{A}_{2,2}, \\
& B_{2}(t)=\tilde{A}_{1} M_{2}+\tilde{A}_{2} M_{1}-M_{1} \tilde{A}_{2,2}-M_{2} \tilde{A}_{1,2}
\end{aligned}
$$

and $\tilde{A}_{1,2}$ and $\tilde{A}_{2,2}$ are the order $\delta$ and order $\varepsilon$ components, respectively, of the linear part of (3.4).

Finally, we make the $\pi$-periodic change of variables

$$
z=w+\delta^{2} N_{1}(t) w+\varepsilon \delta N_{2}(t) w+\varepsilon^{2} N_{3}(t) w+D_{3}(t)[w]^{3}
$$

in order that the resulting equation for $w$ becomes autonomous through terms of order three in $(w, \delta, \varepsilon)$. From Tables 1.1 and 1.2 we obtain the equation for $w$,

$$
\begin{aligned}
w^{\prime}= & \left(\begin{array}{ll}
0 & \theta \\
0 & 0
\end{array}\right) w+\delta\left(\begin{array}{cc}
0 & 0 \\
\bar{\gamma}_{1} & 0
\end{array}\right) z+\varepsilon\left(\begin{array}{cc}
0 & 0 \\
\bar{\gamma}_{2} & \gamma_{3}
\end{array}\right) w \\
& +\delta^{2} B_{1,2} w+\varepsilon \delta B_{2,2} w+\varepsilon^{2} B_{3,2} w \\
& +\tilde{Q}_{3,2}[w]^{3}+O\left((|w|+|\delta|+|\varepsilon|)^{4}\right),
\end{aligned}
$$

in which $B_{1,2}, B_{2,2}$, and $B_{3,2}$ can be evaluated from Table 1.1 and $\tilde{Q}_{3,2}$ from Table 1.2. From Tables 1.1 and 1.2 , we obtain $\left(w_{2} \rightarrow \theta w_{2}\right)$

$$
\begin{aligned}
& w_{1}^{\prime}=w_{2} \\
& w_{2}^{\prime}=\varepsilon_{1} w_{1}+\varepsilon_{2} w_{2}+\alpha w_{1}^{3}+\beta w_{1}^{2} w_{2}
\end{aligned}+O\left((|w|+|\varepsilon|+|\delta|)^{4}\right)
$$

where

$$
\begin{aligned}
\varepsilon_{1} & =\delta \gamma_{1}+\varepsilon \gamma_{2}+\text { quadratic terms in }(\varepsilon, \delta) \\
\varepsilon_{2} & =\varepsilon \gamma_{3}+\text { quadratic terms in }(\varepsilon, \delta) \\
\gamma_{1} & =\frac{2 \Delta^{\prime}(0) \rho}{\pi^{2}} \\
\gamma_{2} & =\gamma_{1}\left[b y y^{\prime}\right] /\left[y^{2}\right] \\
\gamma_{3} & =-\hat{b} \\
\alpha & =\gamma_{1} \cdot\left[b y y^{\prime 3}\right] /\left[y^{2}\right] \\
\beta & =-3\left[b y^{\prime 2}\right]
\end{aligned}
$$

The order-four terms in (3.5) are $\pi$-periodic in $t$. Ignoring these, we obtain the normal form

$$
\begin{aligned}
& u_{1}^{\prime}=u_{2}, \\
& u_{2}^{\prime}=\varepsilon_{1} u_{1}+\varepsilon_{2} u_{2}+\alpha u_{1}^{3}+\beta u_{1}^{2} u_{2} .
\end{aligned}
$$


Note that our assumptions that $\Delta^{\prime}(0) \neq 0$ and $\hat{b} \neq 0$ ensure that the change of variables $(\varepsilon, \delta)$ to $\left(\varepsilon_{1}, \varepsilon_{2}\right)$ is invertible near the origin. We leave to the reader the task of translating our results from the $\left(\varepsilon_{1}, \varepsilon_{2}\right)$ plane back to the $(\varepsilon, \delta)$ plane.

The normal form (3.6) has been exhaustively studied by several authors $[1,4,10,19]$ under the condition

$$
\alpha \cdot \beta \neq 0
$$

which we assume. We follow the treatment of Carr [4] but the reader may also wish to consult Arnold [2].

The results of Carr [4] on the phase portraits of (3.5) in a neighborhood of the origin for the case $\beta<0$ are summarized in Figs. 3.1-3.4 below. The reader may also consult Arnold [2] where a more careful distinction between spiral points and nodes is made. The corresponding results for $\beta>0$ are obtained from the case $\beta<0$ by using the change of variables $w_{2} \rightarrow-w_{2}, \varepsilon_{2} \rightarrow-\varepsilon_{2}, t \rightarrow-t$. Figs. 3.1 and 3.3 give the bifurcation set in the $\left(\varepsilon_{1}, \varepsilon_{2}\right)$ plane for the phase portraits. The bifurcation curves in those figures are rays emanating from the origin, indicating that they have been determined only to the linear approximation (see [2]). These bifurcation curves separate the $\left(\varepsilon_{1}, \varepsilon_{2}\right)$ plane in the vicinity of the origin into disjoint open sets in each of which the phase portraits of (3.6) are topologically similar.

We consider only the more interesting case that $\alpha<0, \beta<0$, leaving the other case to the interested reader. For this discussion it is convenient to imagine beginning with some value of $\left(\varepsilon_{1}, \varepsilon_{2}\right)$ in Region 1 of Figure 3.1 and following a circular path clockwise around the origin in the $\left(\varepsilon_{1}, \varepsilon_{2}\right)$ plane observing the bifurcations as one crosses the various bifurcation curves. In Region 1, the origin is a stable spiral point which undergoes a Hopf bifurcation as one crosses $H 2$. The noteworthy aspect about this stable periodic orbit is that its period is near $2 \pi / \sqrt{\left|\varepsilon_{1}\right|}$, near the bifurcation curve $H 2$. This periodic orbit remains the only stable structure even as the curve $B$ is crossed, whereupon two unstable steady states bifurcate symmetrically from the trivial steady state. This changes on crossing $H_{1}$ $\left(\varepsilon_{2}=(\beta / \alpha) \varepsilon_{1}, \varepsilon_{1}>0\right)$ into Region 4 where the nontrivial steady states become stable, each casting off an unstable Hopf periodic orbit. Approaching the bifurcation curve $L_{1}\left(\varepsilon_{2}=\frac{4}{5}(\beta / \alpha) \varepsilon_{1}\right)$ from above we find the unstable pair of Hopf periodic orbits

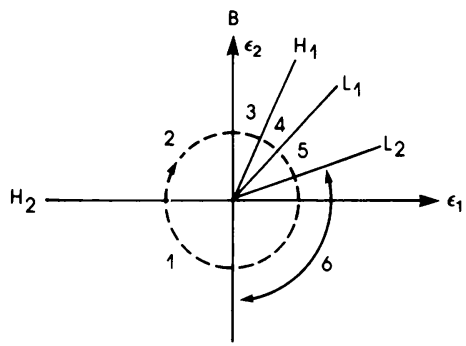

FIG. 3.1. Bifurcation set for $\alpha<0, \beta<0$ 

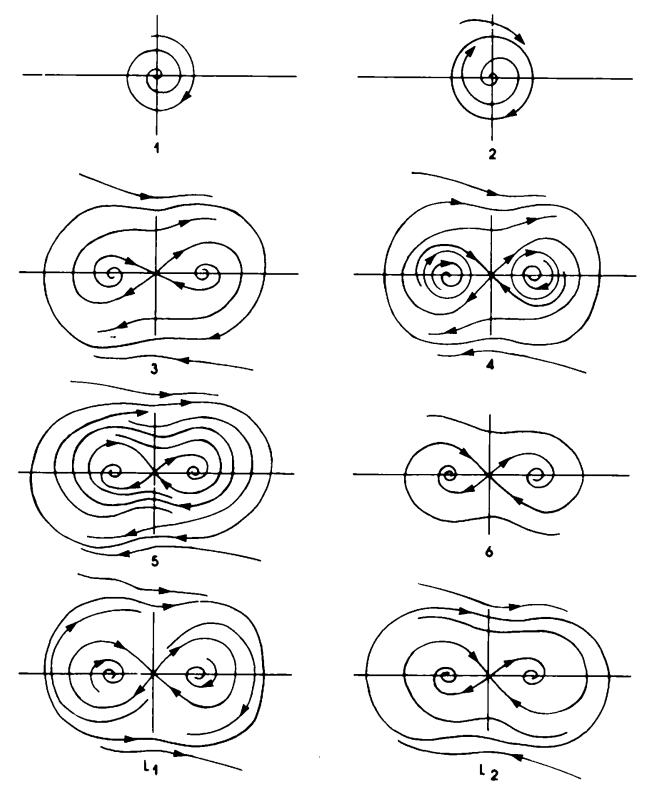

FIG. 3.2. Phase portraits, $\alpha<0, \beta<0$.

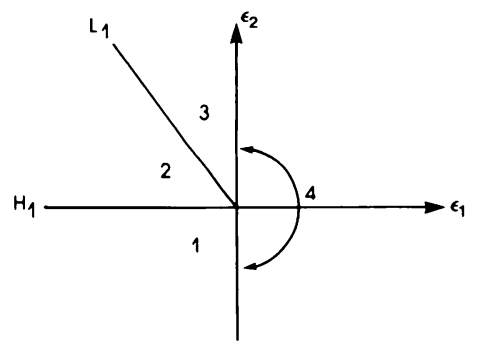

Fig. 3.3. Bifurcation set for $\alpha>0, \beta<0$

coalescing on a figure eight of homoclinic orbits on $L_{1}$. Crossing $L_{1}$, the stable and unstable manifolds of the origin change their orientation and a "large" amplitude unstable periodic orbit is thrown off inside the previously mentioned single Hopf orbit. In Regions 4 and 5 one has the large periodic orbit and the two nontrivial steady states as the only stable structures. On $L_{2}$ the two periodic orbits coalesce and disappear in Region 6 leaving only the stable nontrivial steady states.

Our task at this point is to relate the orbits of the autonomous equation (3.6) to solutions of the periodically perturbed system (3.5). Standard theorems in ordinary differential equations (e.g. [7]) imply that hyperbolic steady states of (3.6) give rise to 

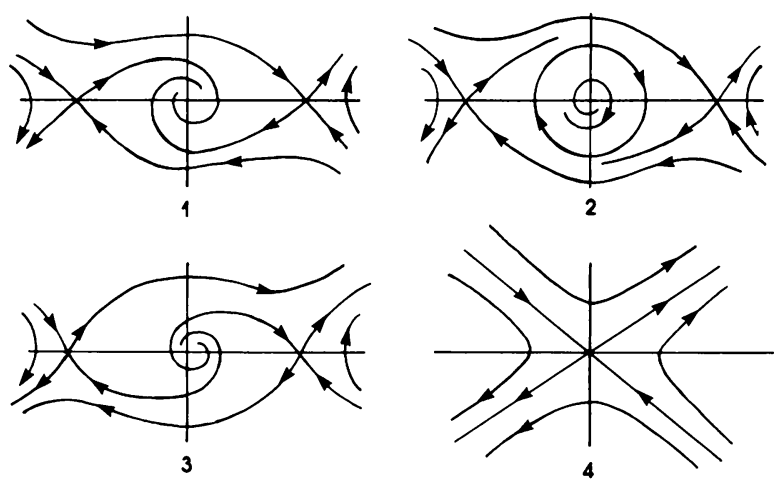

FIG. 3.4. Phase portraits, $\alpha>0, \beta<0$.

hyperbolic $\pi$-periodic solutions of (3.5) with stability types preserved. Similarly, hyperbolic closed orbits of (3.6), viewed as cylinders in $(u, t)$ space, become hyperbolic invariant cylinders in $(w, t)$ space for (3.5) with stability type preserved. In sharp contrast with Section 2 however, the solutions on the various invariant cylinders for (3.5) are very slowly oscillating, for example, the Hopf bifurcation occurring as one crosses the bifurcation curve $H 2$ in Fig. 3.1 has period near $2 \pi / \sqrt{\left|\varepsilon_{1}\right|}$. A similar result is to be expected for the periodic orbits surrounding the nontrivial steady states in Region 4 (these will be stable if $\alpha<0, \beta>0$ ). In the present context, it is unimportant whether or not the rotation number is rational or irrational on an invariant torus since one could not distinguish between solutions which are periodic of period $n \pi$ with $n$ very large and aperiodic slowly oscillating solutions.

In addition to steady states and periodic orbits, Eq. (3.6) has a pair of homoclinic orbits $u_{i}(t), i=1,2$, with $u_{i}( \pm \infty)=0$ for $\left(\varepsilon_{1}, \varepsilon_{2}\right)$ on $L_{1}$ (see Fig. 3.2). The question of whether or not the Poincaré map

$$
P_{\left(\varepsilon_{1}, \varepsilon_{2}\right)}\left(w_{0}\right) \equiv w\left(\pi ; w_{0}, \varepsilon_{1}, \varepsilon_{2}\right),
$$

where $w\left(t ; w_{0}, \varepsilon_{1}, \varepsilon_{2}\right)$ is the solution of (3.5) satisfying $w\left(0 ; w_{0}, \varepsilon_{1}, \varepsilon_{2}\right)=w_{0}$, has homoclinic points near $\Gamma_{i} \equiv\left\{w=u_{i}(t),-\infty<t<\infty\right\}$ for $\left(\varepsilon_{1}, \varepsilon_{2}\right)$ near $L_{1}$ is a delicate one. In the appendix we present arguments which imply that if indeed there are points in $\left(\varepsilon_{1}, \varepsilon_{2}\right)$ space near $L_{1}$ for which homoclinic points exist for $P_{\left(\varepsilon_{1}, \varepsilon_{2}\right)}$, these points occupy a very small region near $L_{1}$. Such a region has the property that for every integer $n$ there is a neighborhood of $(0,0)$ in the $\left(\varepsilon_{1}, \varepsilon_{2}\right)$ plane, depending on $n$, for which the region lying in the neighborhood lies on a family of curves with order of contact $n$ at the origin. Such a region may, of course, have nonempty interior and could be extensive outside a neighborhood of the origin. However, since the resulting chaotic solutions are "chaotic on a slow time scale" (see the scaling in (A2)) they probably could not be distinguished from the long-period subharmonics and slowly oscillating solutions lying on the invariant cylinder. 
3B. $\omega \approx \frac{1}{2}$. In this subsection we consider the resonance $\omega \approx \frac{1}{2}$, that is, when the Floquet multipliers are $\rho \approx \pm i$. Following Sec. $3 \mathrm{~A}$ we will be interested in $(0.3)$ with $a(t)$ given by (0.4) and $\delta=0$ corresponding to $\rho= \pm i$. Our starting point is Eq. (3.2) in which we make the Floquet change of variables (1.2) where $P(t)$ is given by (2.2) in which $\omega=\frac{1}{2}$. The resulting equation becomes

$$
\bar{x}^{\prime}=\frac{1}{2}\left(\begin{array}{cc}
0 & 1 \\
-1 & 0
\end{array}\right) \bar{x}+\varepsilon \tilde{A}_{1}(t) \bar{x}+\delta \tilde{A}_{2}(t) \bar{x}+\tilde{Q}_{3}(t)[\bar{x}]^{3},
$$

in which $\tilde{A}_{1}$ and $\tilde{Q}_{2}(t)[\bar{x}]^{3}$ are given by $(2.5)$ and

$$
\begin{aligned}
& \tilde{A_{2}}=P^{-1} A_{2} P=\mu_{1}\left(\begin{array}{cc}
0 & 1 \\
-1 & 0
\end{array}\right), \\
& \mu_{1}=\frac{1}{2}\left[|y|^{2}\right] .
\end{aligned}
$$

Making a series of changes of variable as in Sec. 3A and using Tables 1.1 and 1.2, we arrive at the normal form

$$
w^{\prime}=\frac{1}{2}\left(\begin{array}{cc}
0 & 1 \\
-1 & 0
\end{array}\right) w+\left[\left(\begin{array}{cc}
\varepsilon \gamma_{1} & \zeta_{1} \varepsilon+\mu_{1} \delta \\
-\zeta_{1} \varepsilon-\mu_{1} \delta & \varepsilon \gamma_{1}
\end{array}\right)+O\left(\varepsilon^{2}+\delta^{2}\right)\right] w+\tilde{Q}_{3,2}(t)[w]^{3},
$$

in which $\tilde{Q}_{3,2}(t)[w]^{3}$ is given in Table 1.2 and $\gamma_{1}$ and $\zeta_{1}$ are given in (2.9).

If we write

$$
w=\left(\begin{array}{l}
w_{1} \\
w_{2}
\end{array}\right) \quad \text { as } w=w_{1}+i w_{2}
$$

then (3.9) becomes

$$
w^{\prime}=\left(\hat{\varepsilon}-\frac{i}{2}\right) w^{\prime}+B e^{-2 i t} \bar{w}^{3}+A|w|^{2} w,
$$

in which

$$
\begin{aligned}
\hat{\varepsilon} & \equiv \varepsilon_{1}+i \varepsilon_{2} \equiv \varepsilon \gamma_{1}-i\left(\zeta_{1} \varepsilon+\mu_{1} \delta\right)+O\left(\varepsilon^{2}+\delta^{2}\right), \\
B & =-\frac{i}{8}\left[b y y^{\prime 3}\right], \\
A & =-\frac{3 i}{8}\left[b\left|y^{\prime}\right|^{2} \bar{y}^{\prime} y\right] .
\end{aligned}
$$

Finally, letting

$$
z=w e^{i t / 2},
$$

the resulting equation for $z$ is autonomous:

$$
z^{\prime}=\hat{\varepsilon} z+B \bar{z}^{3}+A|z|^{2} z .
$$

It should be recalled that the higher-order terms which were dropped from (3.9) are $\pi$-periodic in $t$ and hence the neglected terms in (3.10) are $4 \pi$ periodic in $t$ and $O(|z|+|\hat{\varepsilon}|)^{4}$ uniformly in $t$. We refer to the system in which these terms have not been neglected as the "perturbed (3.10)" in what follows. 
The planar system (3.10) appears to have been first systematically studied by Arnold [1,2] who conjectured many of its possible bifurcations. Since then, Neishtadt [14] has confirmed analytically the conjectures in [1] concerning bifurcation of limit cycles and Berezovskaia and Khibnik have confirmed some conjectures in [3] concerning the "global bifurcations" involving the rearrangement of separatrices, using a combination of analytic and numerical methods. For the latter global bifurcations, a rigorous analysis appears still to be lacking. All work involving (3.10) assumes $B \neq 0$ which we assume to hold. It should be emphasized that all variables and parameters in (3.10) are complex (except for the dependent variable, $t$ ).

In order to describe the various phase portraits for (3.10) it is convenient to do some preliminary scaling of (3.10) to obtain

$$
\dot{\eta}=e^{i \theta} \eta+\tilde{A} \eta|\eta|^{2}+\bar{\eta}^{3}, \cdot=d / d s,
$$

in which $\operatorname{Re} \tilde{A} \leqslant 0$ and $\operatorname{Im} \tilde{A} \leqslant 0$. Table 3.1 below gives the relation between the variables and parameters in (3.10) and those in (3.11). Note the invariance of (3.11) on rotating the $\eta$-plane $90^{\circ}$ about the origin. Also, $\hat{\varepsilon}=r e^{i \phi}$ in Table 3.1 .

TABLE 3.1. $\hat{\varepsilon}=r e^{\prime \phi}$. Note the time reversal in the last two columns.

\begin{tabular}{c|c|c|c|c} 
& $\operatorname{Im} A \leqslant 0$ & $\operatorname{Im} A>0$ & $\operatorname{Im} A \leqslant 0$ & $\operatorname{Im} A>0$ \\
$\operatorname{Re} A \leqslant 0$ & $\operatorname{Re} A \leqslant 0$ & $\operatorname{Re} A>0$ & $\operatorname{Re} A>0$ \\
\hline$s=$ & $\frac{w}{m}, \frac{B \bar{m}^{3}}{r m}=1$ & $\frac{\bar{w}}{\bar{m}}, \frac{B \bar{m}^{3}}{r m}=1$ & $\frac{\bar{w}}{\bar{m}}, \frac{B \bar{m}^{3}}{r m}=-1$ & $\frac{w}{m}, \frac{B \bar{m}^{3}}{r m}=-1$ \\
\hline$\tilde{A}=$ & $r t$ & $r t$ & $-r t$ & $-r t$ \\
\hline$\theta=$ & $\frac{A}{|B|}$ & $\frac{\bar{A}}{|B|}$ & $-\frac{\bar{A}}{|B|}$ & $-\frac{A}{|B|}$ \\
\hline
\end{tabular}

As a result of this scaling we are left with just two independent constants, $\operatorname{Re} \tilde{A}$ and Im $\tilde{A}$, which are both negative. These constants are determined by the function $b(t)$ in $(0.3)$ and the solution $y(t)$ of $(0.2)$ given in (2.1), by the formulae preceding (3.10). It is convenient to consider the bifurcations in the phase portraits for (3.10) for $|\hat{\varepsilon}|$ fixed and small, varying the parameter arg $\hat{\varepsilon}$ so that $\hat{\varepsilon}$ traces out a circle about the origin of small radius. The scaled version (3.11) of (3.10) is ideally suited for this purpose since one simply varies $\theta$ through a range of $2 \pi$.

The sequence of bifurcations in the phase portrait of (3.11) as $\theta$ is varied from 0 to $2 \pi$ for fixed $\tilde{A}$ depends on $\tilde{A}$. In Fig. 3.5 we have indicated some of the regions in the third quadrant of the $\tilde{A}$-plane in which differing sequences of bifurcations are observed. We have not indicated all possible such curves (see [3]). 


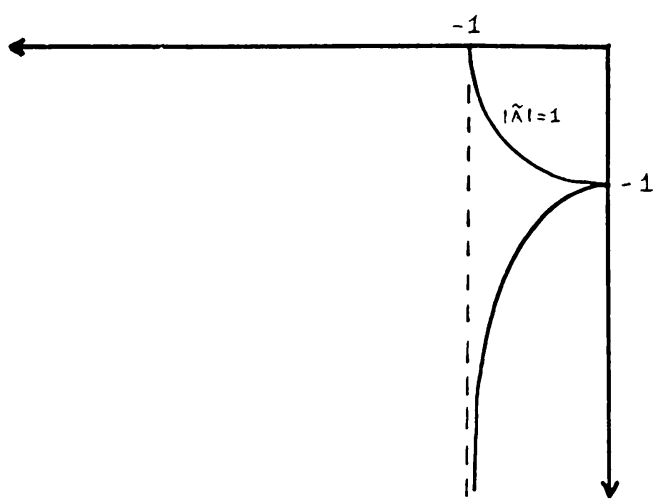

FIG. 3.5. Regions in the 3rd quadrant of $\tilde{A}$-plane.

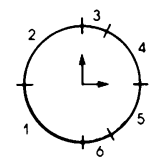

FIG. 3.6. Bifurcation points in case $\operatorname{Re} \tilde{A}<0,|\tilde{A}|<1$.

Perhaps the simplest sequence of bifurcations for (3.11) occurs when $|\tilde{A}|<1$. In this case, the bifurcation points on the unit circle are indicated on Fig. 3.6 and the corresponding phase portraits are shown in Fig. 3.7. Note that in all cases there are four saddle points. The "bifurcation" point separating Regions 1 and 2 simply separates regions where the local rotation around the focus at $z=0$ changes from clockwise to counter-clockwise. There is a Hopf bifurcation at $\theta=\pi / 2$ which is supercritical and stable and this periodic orbit grows, eventually collapsing onto a square consisting of the four saddle points together with saddle-connecting heteroclinic orbits connecting adjacent saddle points, at the point separating Regions 3 from 4 . The sequence of bifurcations in proceeding from Region 4 through 6 are similar to those already described.

The sequence of bifurcations in the phase portrait of (3.11) are also understood in the case that $\operatorname{Re} \tilde{A}<-1$. We have indicated in Fig. 3.8 the bifurcation points on the unit $\left(e^{i \theta}\right)$ circle for the phase portraits and the corresponding phase portraits are sketched in Fig. 3.9. Actually, we have assumed $\operatorname{Im} \tilde{A}<-1$ and that $\tilde{A}$ lies between curves 1 and 2 in Fig. 1 in [3] in the two figures below. In the case $\operatorname{Re} \tilde{A}<-1$ it is possible that the four saddle node pairs in Region 5 occur inside or outside a central cycle which could exist as well in Region 5 (see [3]). In Regions 1 and 2 the origin is globally asymptotically stable. The stability of the origin is lost as Region 3 is entered (or Region 6 from Region 1) and a stable Hopf periodic orbit exists in Region 3 (Region 6). At the bifurcation point 
separating Regions 3 and 4, four degenerate saddle-node points are born on the cycle, promptly separating into four saddle points and four nodes. Each of the saddle point branches of the unstable manifold connect to a node. At the boundary between Regions 4 and 5 the eight critical points coalesce into 4 degenerate saddle-node points and the $d(\arg \eta) / d s=0$ isocline moves inside the periodic orbit which is left by the heteroclinic orbits. On crossing into Region $6, d(\arg \eta) / d s<0$ everywhere in the $\eta$-plane $(\operatorname{Im} \tilde{A}<-1)$.

Describing the metamorphoses of phase portraits of (3.11) when $|\tilde{A}|>1$ and $\operatorname{Re} A>-1$ is far more difficult than the previous cases, being complicated by the possibility that nodes can become foci and change their stability and by the possibility of more complicated separatrices. The curve in Figure 3.5 which asymptotically approaches $\operatorname{Re} \tilde{A}=-1$ as $\operatorname{Im} \tilde{A} \rightarrow-\infty\left(\operatorname{Im} \tilde{A}=-\left[1+(\operatorname{Re} \tilde{A})^{2}\right] / \sqrt{1-(\operatorname{Re} \tilde{A})^{2}}\right.$, see [2]) separates the third quadrant of the $\tilde{A}$-plane into two regions. Only in the right-most region can nodes become foci and change stability type allowing Hopf bifurcations to occur from the peripheral critical points. Rather than attempt a complete description of the possible sequences of bifurcations in the phase portraits of (3.11) in the case $\operatorname{Re} A>-1$ (indeed, it is not clear that a complete picture is as yet rigorously known), we give in Fig. 3.10 a reproduction of Figure 152 in [2] indicating a sequence of bifurcations which is realized for some value of $\tilde{A}$ in this region. Note in particular the four periodic orbits each surrounding a peripheral focus and the "large" closed orbit which exist in 11.

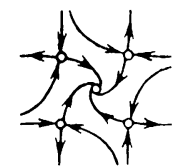

1
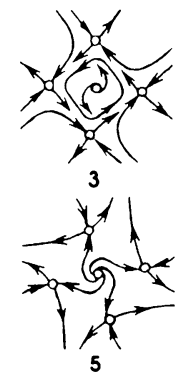
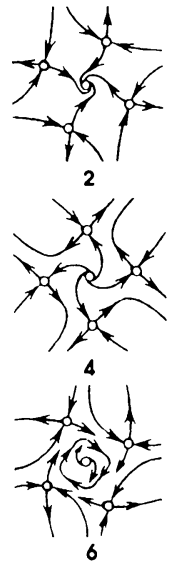

FIG. 3.7. Phase portraits in case $\operatorname{Re} \tilde{A}<0,|\tilde{A}|<1$.

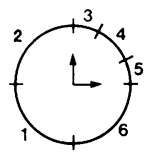

FIG. 3.8. Bifurcation points in case $\operatorname{Re} \tilde{A}<-1, \operatorname{Im} \tilde{A}<-1$. 
The arguments which relate the phase portraits of (3.10) to the solutions of the perturbed (3.10) are identical to those used in $3 \mathrm{~A}$ and we will not dwell on them here. We merely note the possible existence of a stable $4 \pi$-periodic solution occurring together with unstable (saddle-type) $4 \pi$-periodic solution, a "central" integral manifold of slowly oscillating solutions, or peripheral integral manifolds of slowly oscillating solutions surrounding unstable $4 \pi$-periodic solutions for the perturbed (3.10). Homoclinic and heteroclinic points for the Poincaré (period $4 \pi$ ) map are also to be expected but, as in Sec. $3 \mathrm{~A}$, the consequences of their existence may not be distinguishable from the slowly oscillating solutions.

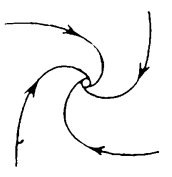

1

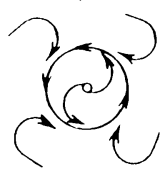

3

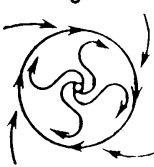

5

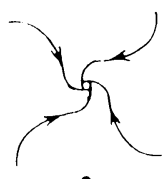

2

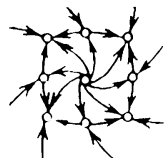

4

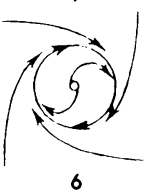

FIG. 3.9. Phase portraits in case $\operatorname{Re} \tilde{A}<-1, \operatorname{Im} \tilde{A}<-1$

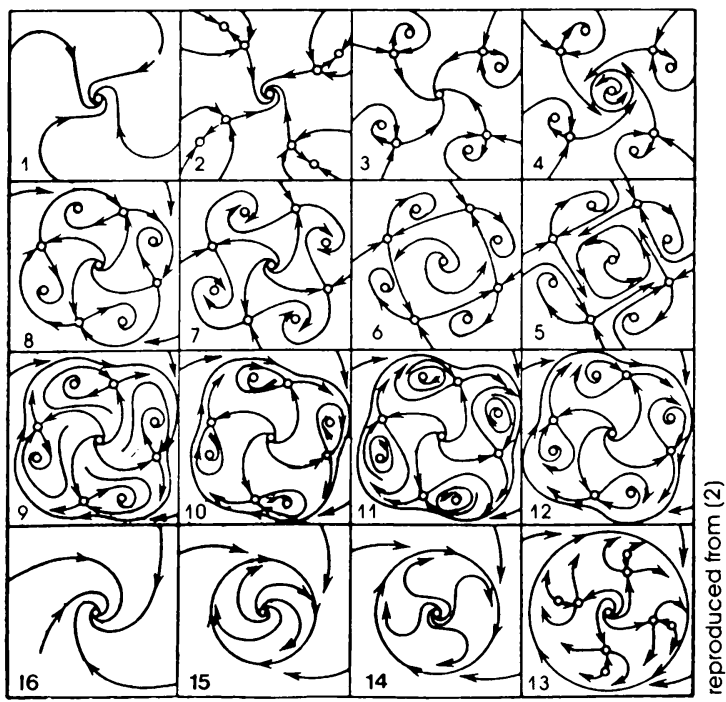

FIG. 3.10. Phase portraits for some $\tilde{A}, \operatorname{Re} \tilde{A}>-1, \operatorname{Im} \tilde{A} \ll-1$ (taken from Figure 152 in [2]). 
Appendix. In this appendix we consider the possible existence of homoclinic points for the Poincare map associated with the trivial saddle point of equation (3.5) for $\left(\varepsilon_{1}, \varepsilon_{2}\right)$ near the curve $L_{1}$ in Fig. 3.1 for the case $\alpha<0, \beta<0$. In [16, 17] we noted that the normal form reduction of an equation of the type (0.3) can be carried out to any finite order $n$ in the variables $\left(x, x^{\prime}, \varepsilon, \delta\right)$ in such a way that the resulting equation is autonomous to order $n$ in these variables. The qualitative form of the resulting equation has been obtained in [6]. If $n=2 k+1$ one obtains

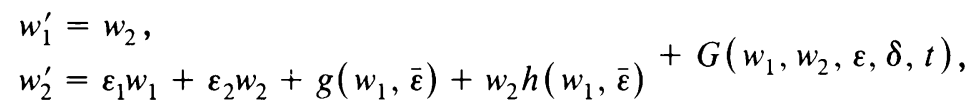

in which

$$
\begin{aligned}
& \bar{\varepsilon}=\left(\varepsilon_{1}, \varepsilon_{2}\right), \\
& \varepsilon_{1}=\delta \gamma_{1}+\varepsilon \gamma_{2}+\text { terms of order at least } 2 \text { but not exceeding } 2 k \text { in }(\varepsilon, \delta), \\
& \varepsilon_{2}=\varepsilon \gamma_{3}+\text { terms of order at least } 2 \text { but not exceeding } 2 k \text { in }(\varepsilon, \delta),
\end{aligned}
$$

with $\gamma_{i}$ as in (3.5). Also

$$
g\left(w_{1}, \bar{\varepsilon}\right)=a_{3}(\bar{\varepsilon}) w_{1}^{3}+a_{5}(\bar{\varepsilon}) w_{1}^{5}+\cdots+a_{2 k+1} w_{1}^{2 k+1}
$$

is a polynomial of degree $2 k+1$ in $\left(\bar{\varepsilon}, w_{1}\right)$ and

$$
h\left(w_{1}, \bar{\varepsilon}\right)=b_{2}(\bar{\varepsilon}) w_{1}^{2}+b_{4}(\bar{\varepsilon}) w_{1}^{4}+\cdots+b_{2 k} w_{1}^{2 k}
$$

is a polynomial of degree $2 k$ in $\left(\bar{\varepsilon}, w_{1}\right)$. Moreover

$$
a_{3}(0)=\alpha \text { and } b_{2}(0)=\beta
$$

as in (3.5). The perturbation term $G$ satisfies

$$
G\left(w_{1}, w_{2}, \varepsilon, \delta, t\right)=G\left(w_{1}, w_{2}, \varepsilon, \delta, t+\pi\right)=O\left[\left(\left|\left(w_{1}, w_{2}\right)\right|+|(\varepsilon, \delta)|\right)^{2 k+2}\right]
$$

uniformly in $t$. In addition, it contains only odd-order terms in $\left(w_{1}, w_{2}\right)$. It should be noted that $\varepsilon_{1}$ and $\varepsilon_{2}$ are not the same $\varepsilon_{1}$ and $\varepsilon_{2}$ defined in (3.5). The latter were polynomials of degree two in $(\varepsilon, \delta)$ while here they are polynomials of degree $2 k$ in $(\varepsilon, \delta)$. The two definitions agree through the order-two terms in $(\varepsilon, \delta)$ however. Similarly, note that (A1) agrees with (3.5) through cubic terms in $(w, \varepsilon, \delta)$.

We are interested in the case $\alpha<0$ and $\beta<0$ as in Fig. 3.1 and for $\left(\varepsilon_{1}, \varepsilon_{2}\right)$ near the curve $L_{1}$ so both $\varepsilon_{1}$ and $\varepsilon_{2}$ are positive. Following [2], we scale variables in (A1) as follows:

$$
\begin{aligned}
& \varepsilon_{1}=\eta^{2}, \eta>0, \\
& \varepsilon_{2}=\mu \eta^{2}, \mu \in R^{+}, \\
& w_{1}=\eta x / \sqrt{-\alpha}, \\
& w_{2}=\eta^{2} y / \sqrt{-\alpha}, \\
& t=\bar{t} / \eta .
\end{aligned}
$$

The resulting equations for $x$ and $y$ are

$$
\begin{aligned}
& \dot{x}=y+\eta^{2 k+1} \bar{G}_{1}(x, y, \eta, \mu, \bar{t} / \eta), \\
& \dot{y}=x-x^{3}+\eta\left(\mu y-\frac{\beta}{\alpha} x^{2} y\right)+\eta^{2} f(x, y, \eta, \mu)+\eta^{2 k} \bar{G}_{2}(x, y, \eta, \mu, \bar{t} / \eta)
\end{aligned}
$$


in which ${ }^{\cdot}=d / d \bar{t}, f$ contains the autonomous terms in (A1) and $\bar{G}_{i}(x, y, \eta, \mu, s)=$ $\bar{G}_{i}(x, y, \eta, \mu, s+\pi)$ is smooth in $(x, y, \eta, \mu), i=1,2$. We follow the Liapunov-Schmidt version of the Melnikov theory as presented in [5] since they assume only boundedness and not periodicity of perturbation terms. Writing (A3) in scalar form, we have

$$
\ddot{x}-x+x^{3}=\eta\left(\mu-\frac{\beta}{\alpha} x^{2}\right) \dot{x}+\eta^{2} f(x, \dot{x}, \eta, \mu)+\eta^{2 k} \bar{G}(x, \dot{x}, \eta, \mu, \bar{t} / \eta)
$$

in which $G$ satisfies the same properties as the $\bar{G}_{i}$ above.

When $\eta=0$, (A4) has a pair of homoclinic orbits given by $\left(\dot{x}_{0}, x_{0}\right)=( \pm \sqrt{2} \operatorname{sech} t$, $\mp \sqrt{2} \operatorname{sech} t \tanh t$ ) (see [6, Sec. 7.3]) which we label as $\Gamma_{ \pm}$. Because of the symmetry we may consider only solutions near $\Gamma_{+}$.

Following [5], (A4) for $\eta \neq 0$, has a homoclinic solution $x(t)=x(\eta, \mu, t)$ with $\operatorname{dist}\left(x(\eta, \mu, t), \Gamma_{+}\right)$small and

$$
x(\eta, \mu, t) \rightarrow 0 \quad \text { as } t \rightarrow \pm \infty
$$

if and only if a bifurcation function $H\left(\eta, \mu, t_{0}\right)$, defined for small $\eta, \mu \in R$ and $0 \leqslant t_{0} \leqslant \pi$ has a zero. $H$ is obtained exactly as in [5] with the exception that the factor $1 / \eta$ in [5] is ignored and $\alpha$ in [5] is replaced by $\eta t_{0}$ here. It follows that $H$ is $\pi$-periodic in $t_{0}$ and, if $b(t)$ is smooth, then $H$ is smooth in $\left(\eta, \mu, t_{0}\right)$. Furthermore, we may write

$$
H\left(\eta, \mu, t_{0}\right)=\eta \int_{-\infty}^{\infty} \dot{x}_{0}(t)\left[\mu-\frac{\beta}{\alpha} x_{0}^{2}(t)\right] \dot{x}_{0}(t) d t+\eta^{2} h_{1}(\eta, \mu)+\eta^{2 k} h_{2}\left(\eta, \mu, t_{0}\right) .
$$

A simple computation shows that the first integral above vanishes for $\mu=4 \beta / 5 \alpha$ (see [6]). In fact, this is precisely the calculation which gives the curve $L_{1}$ in Fig. 3.1. It follows immediately from (A5) and the implicit function theorem that $\eta^{-1} H$ vanishes on a curve given by

$$
\begin{aligned}
\mu & =\bar{\mu}\left(\eta, t_{0}\right)=\mu_{1}(\eta)+\eta^{2 k-1} \mu_{2}\left(\eta, t_{0}\right), \\
\bar{\mu}\left(0, t_{0}\right) & \equiv 4 \beta / 5 \alpha .
\end{aligned}
$$

In terms of the variables $\left(\varepsilon_{1}, \varepsilon_{2}\right)$ we have shown that (A1) has a homoclinic orbit for $\left(\varepsilon_{1}, \varepsilon_{2}\right)$ lying on the one-parameter family of curves given by

$$
\begin{aligned}
& \varepsilon_{1}=\eta^{2} \\
& \varepsilon_{2}=\eta^{2} \mu_{1}(\eta)+\eta^{2 k+1} \mu_{2}\left(\eta, t_{0}\right), 0 \leqslant t_{0} \leqslant \pi .
\end{aligned}
$$

The homoclinic orbit is given, to lowest order, by

$$
w_{1}(t) \approx \frac{\eta}{\sqrt{-\alpha}} x_{0}\left(\eta\left(t+t_{0}\right)\right) \text {. }
$$

The essential point here is that the integer $k>0$ is arbitrary although the region of validity in both the $\left(\varepsilon_{1}, \varepsilon_{2}\right)$ plane and the $\left(w_{1}, w_{2}\right)$ plane of the above results depends on $k$. Since $\mu_{1}(0)=4 \beta / 5 \alpha$ one sees that for each $t_{0}, 0 \leqslant t_{0} \leqslant \pi$, the curve given in (A6) is tangent to the line $\varepsilon_{2}=\frac{4 \beta}{5 \alpha} \varepsilon_{1}$. Moreover, the family of curves (A6), parametrized by $t_{0}$, meet at the origin in the $\left(\varepsilon_{1}, \varepsilon_{2}\right)$ plane, having order of contact increasing to $+\infty$ as $k$ increases indefinitely. The implication is that the set of $\left(\varepsilon_{1}, \varepsilon_{2}\right)$ near 0 for which (A1) has homoclinic points for the corresponding Poincaré map is small. 


\section{REFERENCES}

[1] V. I. Arnold, Loss of stability of self-oscillations close to resonances and versal deformations of equivariant vector fields, Funct. Anal. Appl. 11, 1-10 (1977)

[2] V. I. Arnold, Geometrical methods in the theory of ordinary differential equations, Springer-Verlag, 1983

[3] F. S. Berezovskaia and A. I. Khibnik, On the bifurcation of separatrices in the problem of stability loss of auto-oscillations near 1:4 resonance, P.M.M. 44, 663-667 (1981)

[4] J. Carr, Applications of center manifold theory, Springer-Verlag, 1981

[5] S. N. Chow, J. K. Hale and J. Mallet-Paret, An example of bifurcation to homoclinic orbits, J. Differential Equations 37, 351-373 (1980)

[6] J. Guckenheimer and P. Holmes, Nonlinear oscillations, dynamical systems, and bifurcations of vector fields, Springer-Verlag, New York, 1983

[7] J. K. Hale, Ordinary differential equations, Wiley-Interscience, New York, 1969

[8] M. R. Herman, Mesure de Lebesgue et nombre de rotation, in Geometry and Topology, J. Palis and M. de Carmo (eds.), Lect. Notes in Math., Vol. 597, Springer-Verlag, 271-293 (1977)

[9] P. J. Holmes, Averaging and chaotic motions in forced oscillations, Siam J. Appl. Math. 38, 65-80 (1980)

[10] E. I. Horozov, Versal deformations of equivariant vector fields for the cases of symmetries of order 2 and 3, Trudy Sem. I. G. Petrovskoyo 5, 163-192 (1979)

[11] G. Iooss, Bifurcation of maps and applications, Math. Studies, Vol. 36, North Holland, Amsterdam

[12] W. Magnus and S. Winkler, Hill's equation, Interscience, New York, 1966

[13] V. K. Melnikov, On the stability of the center for time periodic perturbations, Trans. Moscow Math. Soc. 12 , $1-57(1963)$

[14] A. I. Neishtadt, Bifurcations of the phase pattern of an equation system arising in the problem of stability loss of self-oscillations close to 1:4 resonance, P.M.M. 42, 830-840 (1978)

[15] B. Simon, On the genericity of nonvanishing instability intervals, Ann. Inst. H. Poincaré, A 24, 91 (1976)

[16] H. L. Smith, Dynamic bifurcation in a perturbed Hill's equation, preprint

[17] H. L. Smith, Normal forms for periodic systems, preprint

[18] J. J. Stoker, Nonlinear vibrations, Wiley, New York, 1950

[19] F. Takens, Normal forms for certain singularities of vector fields, Ann. Inst. Fourier 23, 163-195 (1973) 